on the scales did not achieve a good correlation between the two scales.

\section{PO-0260C PERTUSSIS EPIDEMIOLOGY IN IZMIR, TURKEY.}

'Z Kurugol, ${ }^{1}$ E Turkoglu, ${ }^{1} \mathrm{G}$ Koturoglu, ${ }^{2} \mathrm{C}$ Sonmez, ${ }^{2} \mathrm{~N}$ Coplu. ${ }^{1}$ Pediatrics, Ege University Faculty of Medicine, Izmir, Turkey; ${ }^{2}$ Hifzisihha, Hifzishha, Ankara, Turkey

\subsection{6/archdischild-2014-307384.913}

Pertussis (whooping cough) is a highly contagious, life-threatening, vaccine-preventable respiratory infection. Adults can infect infants who have not completed their primary immunization schedule. Besides, the infection can be asymptomatic among adults so that the reported cases of pertussis reflect only a fraction of the actual number of the patients in Turkey. The aim of this study is to determine the antibody levels against B. pertussis toxin (PT) and filamentous heamagglutinin (FHA) in ages from 6 months to $\geq 60$ years in Izmir, Turkey. The study population consisted of 400 healthy subjects. A cluster of sample design developed by EPI of the World Health Organization was carried out for the selection of the study population. Anti-PT and antiFHA levels were tested by in-house ELISA in Public Health Institution of Turkey. Anti-PT IgG levels of $<10 \mathrm{EU} / \mathrm{ml}, \geq 10 \mathrm{EU} / \mathrm{ml}$ and $\geq 100 \mathrm{EU} / \mathrm{ml}$ were accepted as non-immun, immune and possible acute/recent infection, respectively. Of the study population $8.5 \%$ had $<10 \mathrm{EU} / \mathrm{ml}, 68.2 \%$ had $10-100 \mathrm{EU} / \mathrm{ml}$, and $23.3 \%$ had $\geq 100 \mathrm{EU} / \mathrm{ml}$ anti-PT IgG antibodies. According to anti-PT IgG antibody levels $23.7 \%$ of the cases were correlated with possible acute/recent infection. The incidence of possible acute/recent infection ( $\geq 100 \mathrm{EU} / \mathrm{ml}$ anti-PT antibodies) was highest among 10-14 and 20-29 years old. The incidence was lowest (18.9\%) among 5-6 years old and increased in the school age and was highest (34.3\%) among 15-19 years old. Although high infant pertussis vaccination coverage in Turkey, our results showed that, pertussis is endemic, particularly in adolescent and adults. Adolescent and adults can be a major reservoir for the disease who haven't completed their primary immunization.

\section{PO-0260d THE USEFULNESS OF AN ANTIMICROBIAL STEWARDSHIP PROGRAM: EIGHT-YEAR TRENDS OF ANTI-INFECTIVE THERAPY IN A TERTIARY PAEDIATRIC HOSPITAL FROM BARCELONA}

${ }^{1} \mathrm{P}$ Soler-Palacin, ${ }^{2} \mathrm{~A}$ Fernandez-Polo, ${ }^{3} \mathrm{MN}$ Larrosa, ${ }^{3} \mathrm{EM}$ Rosello, ${ }^{1} \mathrm{~A}$ Martin-Nalda, ${ }^{1} \mathrm{C}$ Figueras. 'Pediatric Infectious Diseases and Immunodeficiencies Unit, Hospital Universitari Vall d'Hebron. Institut de Recerca Vall d'Hebron. Universitat Autònoma de Barcelona, Barcelona, Spain; ${ }^{2}$ Pharmacy Department, Hospital Universitari Vall d'Hebron. Institut de Recerca Vall d'Hebron. Universitat Autònoma de Barcelona, Barcelona, Spain; ${ }^{3}$ Microbiology Department, Hospital Universitari Vall d'Hebron. Institut de Recerca Vall d'Hebron. Universitat Autònoma de Barcelona, Barcelona, Spain

\subsection{6/archdischild-2014-307384.914}

Background and aims Antimicrobial drug resistance is a serious threat to public health worldwide. Antimicrobial stewardship program (ASP) information related to the paediatric population is scarce. This study assesses the usefulness of ASP instituted in 2005 in our centre.

Methods Retrospective study in a 214 bed-tertiary care paediatric hospital (52\% patients in high-complexity areas), from 2005 to 2012. Variation in admissions, hospital complexity index, mortality rate, bacterial resistance and invasive fungal filamentous infection (IFFI) episodes were recorded. Rates of systemic antibiotics (glycopeptides, aminoglycosides, carbapenems) and intravenous antifungal drugs consumption in admitted patients were assessed, calculated by drug units and related cost.

Results A significant decrease in the number of admissions ($27 \%$ ) was observe but complexity index and number of transplants increased significantly $(+206 \%$ and $+14 \%$, respectively), as episodes of proven and probable IFFI (+88\%). ESBL E.coli and K.pneumoniae increased (5 to $7.6 \%$ and 13.8 to $20 \%$ ) while AmpC hiperproduced Enterobacter cloacae remained stable (29.2 to $29.5 \%)$. Multiresistant P. aeruginosa (1.1 to 5\%) and MRSA (6.5 to $12.2 \%$ ) increased moderatelely. Mortality rate showed a decrease of $8 \%$.

The use of aminoglycosides (-24\%) and glycopeptides (-3\%) decreased while carbapenem and antifungal drug use increased ( +45 and $+76 \%$, respectively) less than complexity indicators along these years. Global antimicrobial cost slightly increased $(+14 \%)$.

Conclusions Since ASP implementation a considerable proportional decrease in anti-infective drugs use in comparison to complexity indexes and severe infection episodes was observed without an increase in mortality. ASP should be implemented in all high-complexity paediatric hospitals to optimise patient's care.

\section{Intensive Care and Paediatric Emergency Care Medicine}

\section{PO-0261 CLINICAL APPLICATION OF BIOMARKERS FOR CHILDREN WITH SEVERE ENTEROVIRUS 71 INFECTION}

${ }^{1} \mathrm{SL}$ Jan, ${ }^{2} \mathrm{SC}$ Chan, ${ }^{1} \mathrm{YC}$ Fu, ${ }^{1} \mathrm{MC}$ Lin. ${ }^{1}$ Department of Pediatrics, Taichung Veterans General Hospital, Taichung, Taiwan; ${ }^{2}$ Department of Nursing, Ta-Jen University, Pingtung County, Taiwan

\subsection{6/archdischild-2014-307384.915}

Background Severe enterovirus 71 (EV71) infection in children can result in high morbidity and mortality. The purpose of this study was to use biomarkers for detection of EV71 infection with cardiac involvement.

Methods A total of 53 children, aged $2.5 \pm 1.7$ years, were studied. Patients were divided into three groups. Group I comprised 30 normal control patients. Group II included 20 patients with EV71-stage 2 infection, and group III included 3 patients with EV71-stage 3 infection. The demographic data, laboratory results and plasma BNP levels were statistically analysed.

Results All group II patients recovered completely without neurological sequelae. Two patients in group III were rescued by ECMO and successfully weaned off and survived without cardiac complications. The group III patients had higher plasma troponin-I, creatine kinase-MB fraction, B-type natriuretic peptide (BNP) level and BNP $\mathrm{z}$-score than those of other groups. The median BNP values were $<5,9.5$ and $238 \mathrm{pg} / \mathrm{mL}$, and median of BNP z-scores were $-2.02,-0.22$, and 6.11 in the three groups, respectively. Using a BNP cut-off value of 100 to identify cases with concomitant severe EV71 infection and acute heart failure, the sensitivity and specificity were $100 \%$ and $100 \%$, respectively. The group III patients had higher urine catecholamine levels than those of group II $(\mathrm{p}<0.01)$.

Conclusions Children with severe EV71 infection have varying degrees of myocardial stress that would be caused by 